\title{
Inter-Modal Wavelength Conversion in Silicon Waveguide
}

\author{
C. Lacava ${ }^{(1, *)}$, M.A. Ettabib(1), T.Dominguez Bucio(1), G. Sharp (2), A.K. Khokhar ${ }^{(1)}$, Y. Jung (1), \\ D.J. Richardson (1), P. Petropoulos ${ }^{(1)}$, F. Gardes ${ }^{(1)}$, M. Sorel(2), F. Parmigiani ${ }^{\left(1,{ }^{* *}\right)}$ \\ (1) Optoelectronics Research Centre, University of Southampton, SO17 1BJ, Southampton (UK) \\ *C.Lacava@soton.ac.uk,**frp@orc.soton.ac.uk \\ (2) School of Engineering, University of Glasgow, G12 8LP, Glasgow (UK)
}

\begin{abstract}
We report the first demonstration of inter-modal wavelength conversion in a silicon waveguide using a telecom-compatible dual-pump CW scheme. Phase-matched inter-modal four-wave-mixing is achieved locating the pumps in the $C$-band and the signal in the L-band, and vice versa.
\end{abstract}

\section{Introduction}

Silicon photonics waveguides, exhibiting large third-order nonlinear effects, at moderate pump powers, are of great interest for the development of functional on-chip all-optical signal processing devices. Various components and devices have already been demonstrated for applications such as wavelength conversion and routing, all-optical switching and format conversion. Due their format-agnostic properties, four wave mixing (FWM) processes are widely used in $\chi^{(3)}$ media to realize signal wavelength conversion. FWM processes require phase matching among the interacting waves; this is typically achieved by tailoring the waveguide geometry to engineer the group velocity dispersion. In this way, impressive demonstrations of broadband operations over a wide wavelength range have been demonstrated using single mode engineered waveguide designs. ${ }^{2}$ However, it is very difficult to simultaneously achieve both features, especially if distinct multi-band operation is desired.

Dispersion engineered multi-mode nonlinear waveguides offer the extra degree of freedom by utilizing a higher number of modes to fulfill the required phase matching and allow for broad-band operation at multiple discrete spectral bands, even at spectral locations far away from the pump wavelength(s). For this reason, inter-modal FWM processes have recently regained interest, primarily driven and supported by developments in space division multiplexing communications which has provided the technology to more precisely excite, analyse and control higher order modes inside the waveguides. ${ }^{4}$ Thus, by properly engineering the modal group velocity profiles within multi mode fibers, researchers have demonstrated the generation and the wavelength conversion of signals for signalto-idler wavelength detunings of up to $700 \mathrm{~nm}$ (in some instances within multiple distinct bands), using both pulsed and (quasi) continuous wave (CW) pumping schemes ${ }^{3,4,5}$ In multi-mode silicon waveguides several FWM-based wavelength conversion demonstrations, in which the interacting waves excite the same mode (intra-modal FWM), have already been carried out, where the absence of phase-matched inter-modal FWM allowed the simultaneous processing of multiple signals in different modes, see for example. ${ }^{6}$ The first preliminary demonstration of inter-modal FWM in a multi mode silicon waveguide was only recently reported, ${ }^{7}$ where FWM between different modes was observed using a high peak power ps-pulse pump source. In this work, we show the realization of a wavelength conversion scheme in a dispersion engineered multi-mode silicon waveguide using CW-pumping. Intermodal FWM-based wavelength conversion of a signal, launched in one spatial mode and located in the L-band (C-band), is achieved using two CW pumps, launched in a different spatial mode and positioned in the C-band (L-band). We believe this represents the first demonstration of a critical step towards the achievement of on-chip tunable discrete wavelength converters for telecommunication applications that can be detuned by hundreds of $\mathrm{nm}$ from the signal/pump wavelengths by exciting phase matched and dispersion tailored modes of a single multi-mode nonlinear waveguide.

\section{Operation principle and experimental set-up}

The four wave configuration of the desired intermodal FWM process and the corresponding operational principle is shown in Fig. 1(a), where it is illustrated how a single device can be made to work across many different frequency bands by exciting different spatial modes. ${ }^{4}$ In the figure the simulated group index curves of the first 
(a)

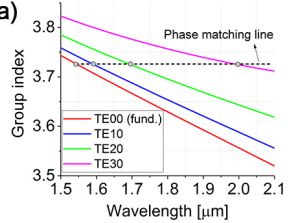

(b)

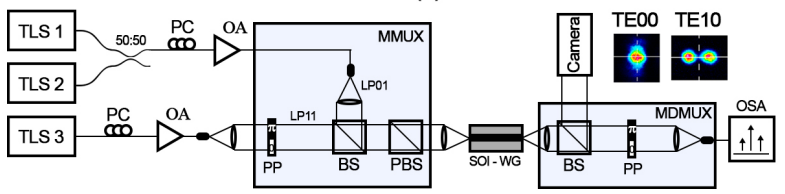

(c)

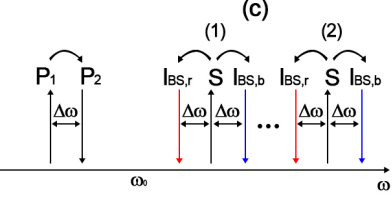

Fig. 1: (a) numerical simulations showing the calculated group index as a function of wavelength for each mode (up to TE30) supported by the waveguide under test; (b) Experimental set-up of the inter-modal FWM wavelength converter; (c) FWM-BS processes.

four supported modes are shown as a function of wavelength for our engineered multi-mode waveguide. Phase matched inter-modal FWM is fulfilled when the propagation constants of the interacting waves satisfy conservation of momentum (as well as conservation of energy). For small pump-to-pump frequency detunings, this occurs when the derivative of the propagation constant, i.e. the inverse group velocity, in one mode is the same as that in an other mode, see dashed black line in Fig.1(a). These numerical results show the possibility of achieving phase matching across multiple discrete wavelength bands, from $1.55 \mu \mathrm{m}$ up to $2 \mu \mathrm{m}$, by exciting the corresponding high order modes. The experimental set-up is shown in Fig. 1(b). Three CW laser sources were used to generate the pump and the signal waves. The co-polarized pumps, $P_{1}$ and $P_{2}$, were launched into the fundamental mode, while the signal wave was launched into the LP11 mode, respectively, using a modemultiplexer (MMUX) based on free-space phase plates (PPs), see Fig. 1(b). The optical beams were coupled to and from the waveguide by using a 40x objective, by means of the end-fire coupling technique. The fundamental mode excited the TE waveguide fundamental mode (TE00), while the LP11 mode excited the TE10 waveguide mode, see corresponding inset images of Fig. 1(b) for their spatial mode distributions after propagation in the waveguide. The de-multiplexer used the same free-space technology used in the mux. The demuliplexer allowed extinction of either the TE00 or the TE10 optical mode, with a measured modal purity of, at least, $10 \mathrm{~dB}$. The output optical spectra were recorded using an optical spectrum analyzer (OSA). To measure intra-modal FWM we removed the PPs at the input and output of the set-up to ensure that all the signal waves were exciting only the fundamental mode of the waveguide which was then coupled into the SM output fiber.

\section{Experimental results}

We first characterized the linear and nonlinear behaviour of our SOI sample (waveguide length 7 $\mathrm{mm})$. Propagation losses were measured to be $1.5 \mathrm{~dB} / \mathrm{cm}$ for both the TE00 and TE10 modes. We estimated a coupling loss of $7.6 \mathrm{~dB} /$ facet and $8.1 \mathrm{~dB} /$ facet for the TEO0 and TE10 modes, respectively. The waveguide nonlinear parameter (TE00 mode) was measured to be $8(\mathrm{Wm})^{-1}$. We operated the wavelength converter using only the first two modes of the SOI waveguide: TE00 and TE10 (exhibiting an inter-modal nonlinear modal overlap of about $70 \%$ ). Following the outcome of our numerical simulations (see Fig. 1(a)) to fulfill inter-modal phase matching, we placed the two pump signals at wavelengths $\lambda_{p 1}=1544.75$ $\mathrm{nm}$ and $\lambda_{p 2}=1545.25 \mathrm{~nm}$, respectively, while the signal was positioned at $\lambda_{s}=1608.7 \mathrm{~nm}$. In Fig. 2(a) we show an example of the signal and the generated idler spectra for the intra- and intermodal FWM processes, respectively. The measured inter-modal FWM efficiency was $-35 \mathrm{~dB}$ (blue line in Fig. 2(a)), while the intra-modal FWM was $-45 \mathrm{~dB}$ (red line in Fig. 2(a)). This 10 $\mathrm{dB}$ improved conversion efficiency demonstrates that phase matching was achieved for the intermodal process when the pumps-to-signal detuning was $70 \mathrm{~nm}$, while only partial phase matching was observed for the intra-modal process. A second set of measurements was performed in order to assess the bandwidth of the proposed wavelength conversion scheme. In Fig. 2(b) we show the normalized conversion efficiency as a function of the signal wavelength, when the two pump beams were kept fixed at $\lambda_{p 1}=1544.75 \mathrm{~nm}$ and $\lambda_{p 2}=1545.25 \mathrm{~nm}$, for both the inter and intramodal FWM processes (blue and red dots, respectively). In the inter-modal configuration, the phase matching condition was no longer fully satisfied when the signal was detuned from the starting wavelength of $\lambda_{s}=1608.7 \mathrm{~nm}$, and as a consequence we observed a drop in terms of conversion efficiency. $\mathrm{A} 3 \mathrm{~dB}$ efficiency penalty was measured when the signal wavelength was detuned by $9 \mathrm{~nm}$ from the phase matched wavelength towards shorter wavelengths. This measurement was also performed to characterize the intra-modal FWM configuration (Fig. 2(b). red 


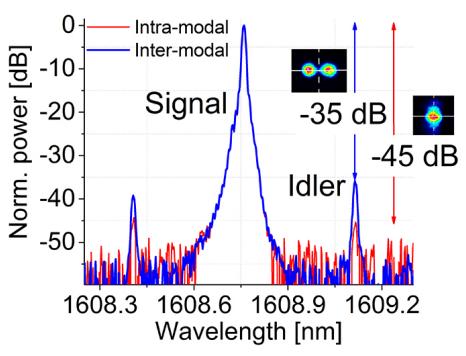

(a)

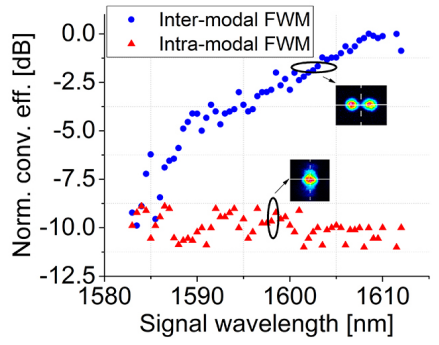

(b)

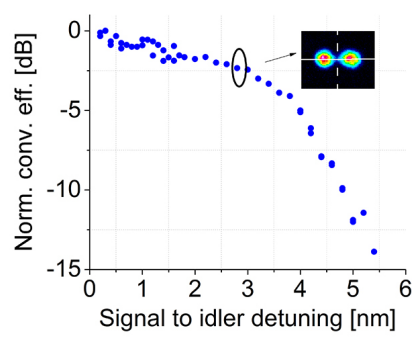

(c)

Fig. 2: (a) Examples of typical spectra at the output sample after inter- (blue curve) and intra- (red curve) modal FWM processes when the signal wavelength is of $1608.67 \mathrm{~nm}$. (b) normalized conversion efficiency as a function of the signal wavelength for the inter-modal (blue dots) and intra-modal (red-triangles) FWM processes, respectively, (c) normalized conversion efficiency as a function of the signal to idler detuning for the inter-modal FWM process only.

triangles). In this case, the FWM efficiency was relatively constant as a function of signal wavelength due to the short length of the sample (i.e. showing low overall dispersion), with a small increase of efficiency as the signal is tuned closer to the pump wavelengths as the process becomes better phase matched. The inter-modal phase matching bandwidth was also assessed by measuring the FWM-efficiency as a function of the signal to idler detuning. Measurements were taken by gradually detuning the wavelength of one pump whilst keeping the other pump wavelength fixed $\left(\lambda_{p 1}=1544.75 \mathrm{~nm}\right)$. The results are reported in Fig. 2(c), revealing a $-3 \mathrm{~dB}$ half-bandwidth of $4 \mathrm{~nm}$. Much broader bandwidths are to be expected by engineering the group index of the two spatial modes such that they are shifted replicas of one another at the wavelengths of interest. ${ }^{4}$ We have repeated similar measurements by swapping the location of the pumps and signalidler wavelengths to demonstrate, i.e. locating the pumps in the L-band and the signal-idler pair in the C-band and obtained comparable results, not shown here. In silicon, the FWM-efficiency is mainly limited by two photon absorption and free carrier effects. ${ }^{1}$ For this reason, increasing the pump power and/or the length of the waveguide, would not significantly increase the FWMefficiency, when a CW-pumps scheme is considered. In order to substantially increase the FWM-efficiency we have recently designed and fabricated an inter-modal wavelength converted based on our internally developed silicon-rich silicon nitride platform. ${ }^{8}$ Preliminary results, not reported here, have shown the potential to achieve higher efficiency, with pump powers $<500 \mathrm{~mW}$.

\section{Conclusion}

We have demonstrated a dual- pump CW silicon photonics wavelength converter, using an intermodal FWM process. We report a conversion efficiency of $-35 \mathrm{~dB}$, between the TE00 and the TE10 waveguide modes, when a pump power of 120 $\mathrm{mW}$ is coupled into the waveguide, with a pumpsto-signal detuning of about $70 \mathrm{~nm}$. Preliminary results in the silicon-rich silicon nitride platform suggest the potential of much higher conversion efficiencies. Our simulated group index curves predict multiple phase matching bands, spanning from $1.5 \mu \mathrm{m}$ to $2 \mu \mathrm{m}$, if the signal/pumps are used to excite higher order modes of the same multimode waveguide.

\section{Acknowledgements}

This work was supported by the EPSRC through the Project SPFS and EP/P026575/1, EP/R003076/1 and EP/L021129/1 grants. http://doi.org/10.5258/SOTON/D0425

\section{References}

[1] C. Lacava et al., "Nonlinear Silicon Photonic Signal Processing Devices for Future Optical Networks," Applied Sciences, vol. 7, no. 1, p. 103, 2017.

[2] M. Foster et al., "Broad-band optical parametric gain on a silicon photonic chip.," Nature, vol. 441, no. 7096 , pp. 960-963, 2006.

[3] J. Demas et al., "Broadband and Wideband Parametric Gain via Intermodal Four-Wave Mixing in Optical Fiber," Conference on Lasers and Electro-Optics, no. 2, SM3M.1, 2017.

[4] F. Parmigiani et al., "All-optical mode and wavelength converter based on parametric processes in a threemode fiber," Optics Express, vol. 25, no. 26, p. 33602 , 2017.

[5] R. Essiambre et al., "Experimental Investigation of InterModal Four-Wave Mixing in Few-Mode Fibers," IEEE Photon. Technol. Lett., vol. 25, no. 6, pp. 539-542, 2013.

[6] Y. Ding et al., "Mode-selective wavelength conversion based on four-wave mixing in a multimode silicon waveguide," Optics Express, vol. 22, no. 1, p. 127, 2014, ISSN: 1094-4087.

[7] S. Signorini et al., "Broad wavelength generation and conversion with Multi Modal Four Wave Mixing in sili- con waveguides," in Group IV Photonics, 2017, pp. 59-60, ISBN: 9781509065677.

[8] C. Lacava et al., "Si-rich Silicon Nitride for Nonlinear Signal Processing Applications," Scientific Reports, vol. 7, no. 1, 2017. 